\title{
Novel Ti-O-Ti Bonding Species Constructed in a Metal-Oxide \\ Cluster. Reaction Products of Bis(oxalato)oxotitanate(IV) with the Dimeric, 1,2-Di-Titanium(IV)-Substituted Keggin Polyoxotungstate
}

Kunihiko Hayashi, Hideyuki Murakami and Kenji Nomiya*

Department of Materials Science, Faculty of Science, Kanagawa University, Hiratsuka, Kanagawa 259-1293, Japan

\section{Contents:}

Fig. S1 Temperature-dependent ${ }^{31} \mathrm{P}$ NMR of 1 in $\mathrm{D}_{2} \mathrm{O}$ : (a) at $23.2^{\circ} \mathrm{C}$, (b) at $49.7{ }^{\circ} \mathrm{C}$, (c) at $79.7{ }^{\circ} \mathrm{C}$, and $(\mathrm{d})$ the solution of $(\mathrm{c})$ remeasured at $23.0^{\circ} \mathrm{C}$.

Table S1 Average bond distances $(\AA)$ and angles $\left(^{\circ}\right)$ [range] for the Keggin POM moiety in 1a

Table S2 Bond valence sum (BVS) calcutions of W(1-10), Ti(1-4), P and O atoms 


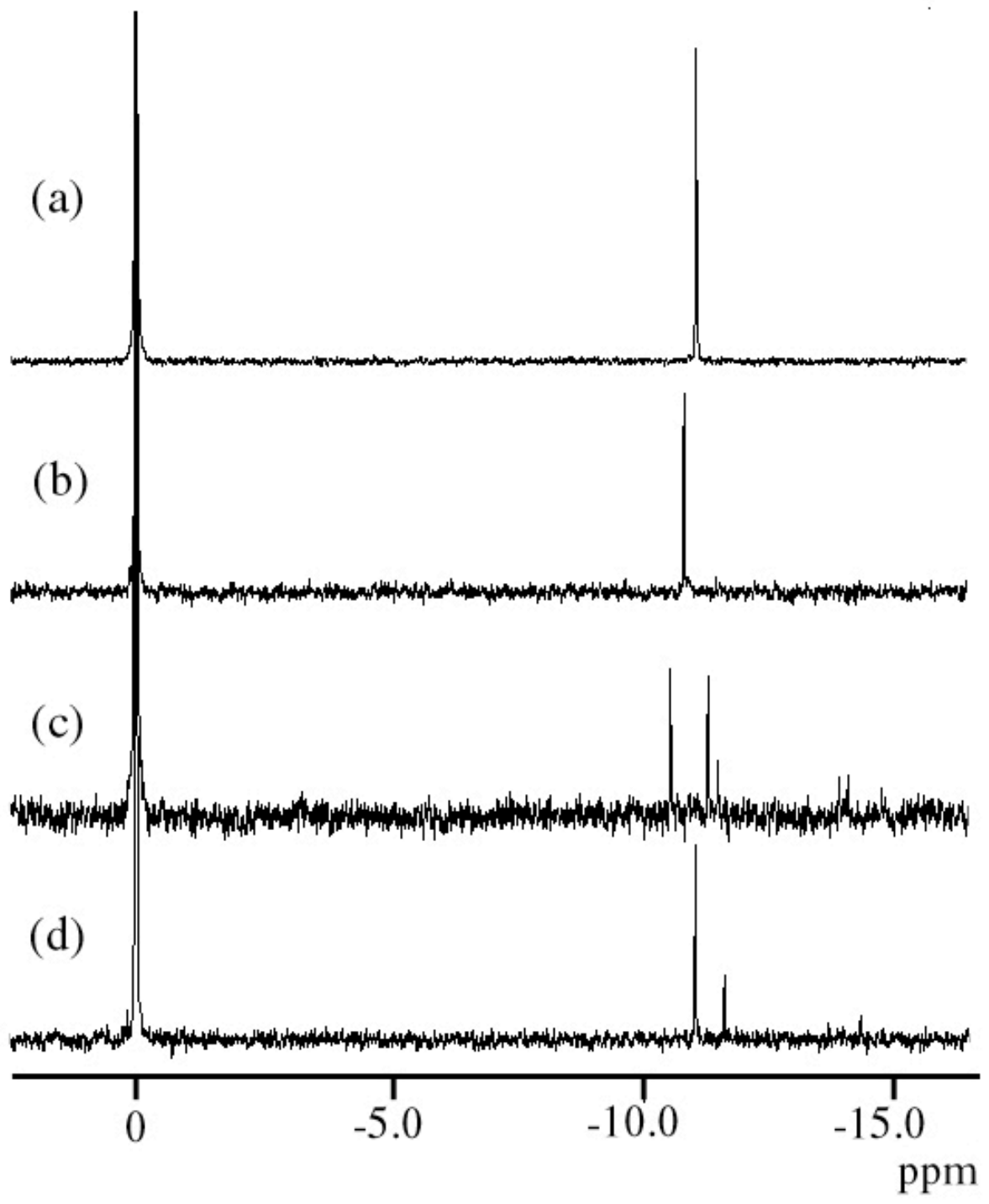

Fig. S1 
Table S1 Average bond distances $(\AA)$ and angles $\left(^{\circ}\right)$ [range] for the Keggin POM moiety in $1 \mathbf{a}$

W-Ot (Ot : terminal oxygen)

1.710 [1.682(12) - 1.723(10)]

$\mathrm{W}-\mathrm{Oc}$ (Oc : corner sharing oxygen)

$1.907[1.862(10)-1.969(10)]$

W-Oe (Oe : edge-sharing oxygen)

$1.921[1.872(10)-1.979(11)]$

$\mathrm{W}-\mathrm{Oa}(\mathrm{Oa}$ : oxygen coordinated to $\mathrm{P}$ atom $) \quad 2.407$ [2.335(10) - 2.473(10)]

$\mathrm{W}-\mathrm{Ob}(\mathrm{Ob}:$ oxygen coordinated to Ti atom) $1.870[1.854(11)-1.897(10)]$

P-O distance $\quad 1.539[1.517(10)-1.559(10)]$

O-P-O angles $109.5[106.8(5)-111.6(6)]$ 
Table S2 Bond valence sum(BVS) calcutions of W(1-10), Ti(1-4), P and O atoms

\begin{tabular}{|c|c|c|c|c|c|}
\hline $\mathrm{W}(1)$ & 6.174 & \multicolumn{2}{|c|}{$\mathrm{W}(6)$} & \multicolumn{2}{|l|}{6.104} \\
\hline $\mathrm{W}(2)$ & 6.288 & \multicolumn{2}{|c|}{$\mathrm{W}(7)$} & \multicolumn{2}{|l|}{6.205} \\
\hline $\mathrm{W}(3)$ & 6.148 & \multicolumn{2}{|c|}{$\mathrm{W}(8)$} & \multicolumn{2}{|l|}{6.189} \\
\hline $\mathrm{W}(4)$ & \multicolumn{2}{|l|}{6.299} & $\mathrm{~W}(9)$ & \multicolumn{2}{|l|}{6.083} \\
\hline $\mathrm{W}(5)$ & \multicolumn{2}{|l|}{6.180} & $\mathrm{~W}(10)$ & \multicolumn{2}{|l|}{6.161} \\
\hline \multicolumn{6}{|c|}{$\mathrm{W}(1-10)$ average $=6.183[6.083-6.299]$} \\
\hline $\operatorname{Ti}(1)$ & \multicolumn{2}{|l|}{4.294} & $\operatorname{Ti}(3)$ & \multicolumn{2}{|l|}{4.278} \\
\hline $\operatorname{Ti}(2)$ & 4.182 & \multicolumn{2}{|c|}{$\operatorname{Ti}(4)$} & \multicolumn{2}{|l|}{4.252} \\
\hline \multicolumn{6}{|c|}{$\operatorname{Ti}(1-4)$ average $=4.252[4.182-4.294]$} \\
\hline $\mathrm{P}(1)$ & 4.947 & & & & \\
\hline $\mathrm{O}(1)$ & 2.145 & $\mathrm{O}(16)$ & 1.726 & $\mathrm{O}(31)$ & 2.058 \\
\hline $\mathrm{O}(2)$ & 1.731 & $\mathrm{O}(17)$ & 2.023 & $\mathrm{O}(32)$ & 1.708 \\
\hline $\mathrm{O}(3)$ & 2.113 & $\mathrm{O}(18)$ & 1.731 & $\mathrm{O}(33)$ & 1.918 \\
\hline $\mathrm{O}(4)$ & 1.984 & $\mathrm{O}(19)$ & 2.089 & $\mathrm{O}(34)$ & 1.712 \\
\hline $\mathrm{O}(5)$ & 2.051 & $\mathrm{O}(20)$ & 1.812 & $\mathrm{O}(35)$ & 1.915 \\
\hline $\mathrm{O}(6)$ & 2.104 & $\mathrm{O}(21)$ & 2.032 & $\mathrm{O}(36)$ & 1.689 \\
\hline $\mathrm{O}(7)$ & 2.101 & $\mathrm{O}(22)$ & 1.873 & $\mathrm{O}(37)$ & 2.027 \\
\hline $\mathrm{O}(8)$ & 2.059 & $\mathrm{O}(23)$ & 1.884 & $\mathrm{O}(1 \mathrm{H})$ & 0.420 \\
\hline $\mathrm{O}(9)$ & 1.925 & $\mathrm{O}(24)$ & 1.907 & $\mathrm{O}(2 \mathrm{H})$ & 0.298 \\
\hline $\mathrm{O}(10)$ & 1.887 & $\mathrm{O}(25)$ & 1.963 & $\mathrm{O}(3 \mathrm{H})$ & 0.390 \\
\hline $\mathrm{O}(11)$ & 2.104 & $\mathrm{O}(26)$ & 2.131 & $\mathrm{O}(4 \mathrm{H})$ & 0.488 \\
\hline $\mathrm{O}(12)$ & 1.717 & $\mathrm{O}(27)$ & 1.956 & $\mathrm{O}(1 \mathrm{M})$ & 1.947 \\
\hline $\mathrm{O}(13)$ & 2.030 & $\mathrm{O}(28)$ & 1.949 & $\mathrm{O}(2 \mathrm{M})$ & 2.078 \\
\hline $\mathrm{O}(14)$ & 1.783 & $\mathrm{O}(29)$ & 2.034 & $\mathrm{O}(3 \mathrm{M})$ & 1.998 \\
\hline $\mathrm{O}(15)$ & 2.116 & $\mathrm{O}(30)$ & 1.971 & & \\
\hline
\end{tabular}

